\title{
Le clair-obscur
}

\section{Frosa Pejoska-Bouchereau}

\section{(2) OpenEdition}

1 Journals

Édition électronique

URL : https://journals.openedition.org/ceb/1475

DOI : $10.4000 /$ ceb. 1475

ISSN : 2261-4184

Éditeur

INALCO

Édition imprimée

Date de publication : 1 décembre 2008

Pagination : iii-xii

ISBN : 978-2-85831-173-6

ISSN : 0290-7402

Référence électronique

Frosa Pejoska-Bouchereau, "Le clair-obscur », Cahiers balkaniques [En ligne], 36-37 | 2008, mis en ligne le 14 mai 2012, consulté le 06 juillet 2021. URL : http://journals.openedition.org/ceb/1475 ; DOI :

https://doi.org/10.4000/ceb.1475

Ce document a été généré automatiquement le 6 juillet 2021.

\section{c) (i) (3)}

Cahiers balkaniques est mis à disposition selon les termes de la Licence Creative Commons Attribution - Pas d'Utilisation Commerciale 4.0 International. 


\section{Le clair-obscur}

\section{Frosa Pejoska-Bouchereau}

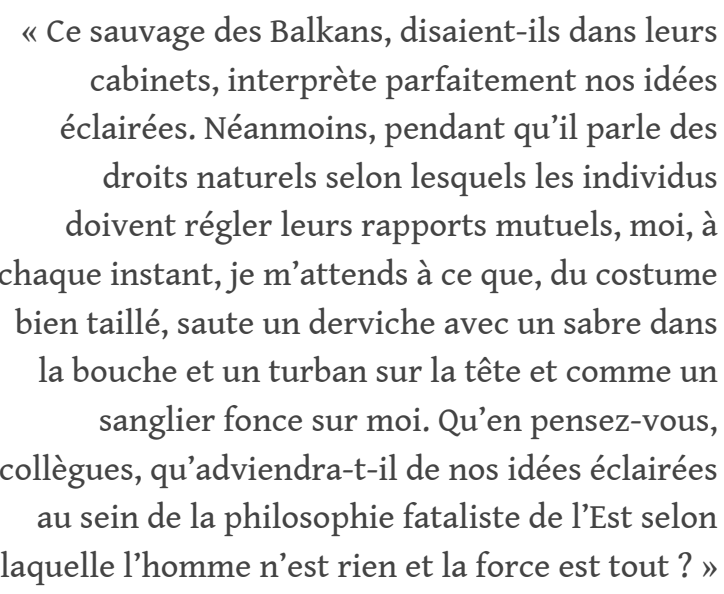

Dans la nouvelle Obscur vilayet de Zoran Kovačeski, Eftim Robe, un étudiant macédonien en philosophie, à la Sorbonne, à la fin du XIX siècle, n'est pas reconnu par les disciples des encyclopédistes malgré ses brillants résultats et sa parfaite maîtrise des idées des Lumières. Les disciples de la « Nouvelle pensée européenne » ne le nommeront pas au poste d'assistant à la chaire de philosophie des encyclopédistes, lui préférant un étudiant français « de beaucoup plus faible et par la pensée et par le savoir que le Slave macédonien ». Lui qui avait pensé appartenir à l'Europe, et participer de l'universel, se voit ramené à une origine, pour ainsi dire à l'origine, quand l'homme se résumait à la force.

2 L'intérêt ${ }^{1}$ pour l'Orient oscille entre admiration et rejet ou encore confortation de l'existence d'une humanité à laquelle nous appartiendrions par comparaison avec cet autre monde de la «Barbaresque». Si l'Orient, en particulier l'Empire ottoman, a toujours fasciné la littérature, en témoignent les nombreux romans et études, nous ne savons rien de sa représentation dans les littératures balkaniques. Mais savons-nous plus de choses sur ces littératures considérées elles-mêmes comme orientales? Les Balkans, et conséquemment les littératures balkaniques, ont revêtu ces mêmes qualificatifs qui étaient attribués à l'Empire ottoman: obscurité, immobilisme, stagnation, domination, oppression, guerre, malheur et désolation et, enfin, génocide. 
Ce «Moyen Age » ottoman, qui dura cinq siècles, marque les Balkans du sceau de son orientalisme. Impossible donc de dissocier littérature balkanique et monde oriental. Or, comment se voient ces littératures en regard de cet Empire, duquel elles ont émergé après sa chute, en tant que représentantes de nouveaux Etats nationaux ?

3 Nous avons tenté de répondre à cette question à travers les contributions de nombreux spécialistes des littératures balkaniques sans oublier d'y associer les spécialistes des pays qui ont partagé pour un temps la même histoire ou on dû lutter contre les campagnes expansionnistes des Ottomans.

En ouverture de ce dossier, Predrag MATVEJEVIĆ procède à l'esquisse des Balkans en des touches nuancées afin de nous permettre d'appréhender la complexité de cet espace, plus difficile à définir qu'à délimiter. Les Balkans, à la pluralité et à la variété démographiques, sont au centre du questionnement en termes de rapports ethniques, de constructions identitaires et territoriales, fondées sur des revendications qui s'appuient aussi bien sur le mythe que sur l'histoire. La fin des Empires et l'avènement de l'Etat-Nation constituent dans ces régions de multiples fractures divisant Églises et croyances, Empires/Etats et pouvoirs, styles et écritures. D'où un riche patrimoine littéraire d'une extraordinaire vivacité.

5 Sineva BENE-KATUNARIĆ souligne la prodigieuse pérennité de la thématique ottomane dans la littérature croate par l'étude des données historiques et leur esthétisation chez trois grands auteurs: Marko Marulić (1450-1524), Ivan Gundulić (1589-1638), Ivan Mažuranić (1814-1891). Elle montre que, grâce à cette thématique, «le pays déchiqueté » et les auteurs dispersés dans différents États pouvaient se réunir dans l'espace littéraire ; la littérature devenant le lieu symbolique de la patrie rêvée.

6 Le territoire peuplé par les Slovènes, possession héréditaire des Habsbourg, ne s'est jamais trouvé sous la domination ottomane. Les incursions des armées ottomanes et des groupes armés $\left(\mathrm{du} \mathrm{XV}^{\mathrm{e}}\right.$ au XVII $\left.{ }^{\mathrm{e}}\right)$ sont les seuls contacts avec les Ottomans. Aussi les Slovènes n'avaient-ils aucune réelle connaissance de leur culture. Si le plus grand nombre des écrits traitant des incursions des Ottomans sont dus aux moines, et donc écrits en latin, le reste étant en allemand, c'est la prose, en slovène, qui se développe durant la seconde moitié du XIX siècle, qui initiera le « roman turc» (turška povest), le genre le plus populaire en Slovénie jusqu'aux débuts du $\mathrm{XX}^{\mathrm{e}}$ siècle et au-delà. Le « roman turc » était publié, presque exclusivement, par la Mohorjeva družba dirigée par les prêtres et qui avait des souscripteurs dans tout le pays. Ces romans se prêtaient à la scolarisation obligatoire des milieux simples dont il s'agissait de satisfaire le goût, ainsi qu'à la nécessité de développer chez le public une conscience d'appartenance nationale, doublée d'une conscience d'appartenance religieuse. À travers cette fiction et la lutte commune contre les Ottomans, il s'agissait de souder la communauté nationale et de l'ancrer dans la sphère de la chrétienté occidentale. Il existe plus d'une trentaine d'œuvres inspirées par ce thème et l'image que le grand public slovène a de cette époque ne vient pas de l'Histoire en tant que telle, mais de la littérature, explique Antonia BERNARD. La thématique turque sert à souligner le contraste entre les Slovènes qui apparaissent dans toute leur splendeur: guerriers et défenseurs courageux, qui n'hésitent pas à tuer, mais uniquement les hommes en armes et, sur fond sombre, les Turcs inhumains et sanguinaires fanatisés par leur foi à combattre.

7 Dans ce genre, le thème du «janissaire » occupe une place de choix. Jurij Kozjak, le janissaire slovène (1864) est l'œuvre du premier romancier slovène Josip Jurčič (1844-1881). Réédité 18 fois avant la fin du XIX ${ }^{\mathrm{e}}$ siècle, le roman fut traduit en 43 
langues, dont le français, au XXe siècle. Cette traduction réalisée par un prête, le père Kolednik, fut couronnée par l'Académie française et connut quatre rééditions. Rado Murnik (1870-1932) publia en feuilleton de 1911 à 1913 Le beau janissaire, un autre «roman turc » célèbre. Anton Aškerc (1856-1912), un prête catholique très intéressé par l'Orient proche et lointain, l'orthodoxie, l'islam, ou le bouddhisme, reprend ce thème dans son célèbre poème « Le janissaire ".

8 "La "Turquerie" est un genre, une figure de style, un motif reconnaissable autant en décor qu'en peinture, qu'en musique ${ }^{2} »$. Que l'Occident ait retenu dans sa musique ce genre marquant n'a rien d'étonnant pour Marc Leuridan, car « Le premier contact entre armées occidentale et turque était musical, les janissaires avançant d'un pas endiablé à la suite de leur clique ${ }^{3}$. " En effet, le «Janissaire » est un terme qui désigne un registre du pianoforte, généralement viennois. Le pianoforte viennois comporte souvent un pédalier fourni d'accessoires qui peuvent modifier les cordes en se posant dessus. Le "Janissaire", « en revanche est indépendant des cordes. Le mécanisme de janissaire est composé de clochettes, d'une cymbale et d'un tambour ${ }^{4} . »$ La Marche turque, de même que L'Enlèvement au Sérail, de Mozart sont des œuvres qui utilisent les percussions de janissaire. Si de la cause à l'effet se dessine le lien logique de ce vestige de l'époque ottomane, il n'en va pas de même de la surprenante adéquation de la technicité du mécanisme du janissaire avec l'état de janissaire ottoman. Indépendant des cordes, le «janissaire du pianoforte" rappelle le «janissaire», levé lors du ramassage de l'impôt du sang, le devchirme, et emmené loin de son lieu d'origine et de sa famille afin de servir l'Empereur et l'Empire : il est celui qui n'a plus de liens. Dans les littératures balkaniques, ce phénomène occupe une place prépondérante par la récurrence de son traitement. À travers deux écrivains macédoniens : Luan Starova et Stale Popov, Frosa PEJOSKA-BOUCHEREAU questionne les raisons de l'importance de cette thématique. Loin de se limiter au seul homo balkanicus, elle tente de montrer - à travers la théorie inédite d'Ernest Gellner, fondée sur le janissariat et le concept de castration dans son ouvrage Nations et Nationalisme, ainsi qu'avec Georges Orwell et sa magistrale fable antitotalitaire : La ferme des animaux, et, enfin, avec Clarissa Henry, Marc Hillel et Léon Poliakov traitant du rapt des enfants et la naissance des «janissaires du III ${ }^{e}$ Reich »-, que ce sujet concerne l'humain dans son humanité.

La représentation du Turc sous les traits de l'ennemi barbare et infidèle est, pour les Russes, le résultat de récits de cruautés, ayant pour cadre les Balkans, relayés par la presse, les brochures de propagande, les correspondances et les mémoires. Pendant plus de deux ans, la population russe subit une surenchère d'images et de récits qui ont la violence pour thème majeur et la justification du conflit pour objectif. Aussi l'image $\mathrm{du}$ Turc, pour les Russes est-elle indissociable de l'imaginaire lié aux Balkans. Pour comprendre la mobilisation patriotique des Russes en faveur de la libération des Slaves du Sud au cours de la crise balkanique et de la guerre russo-turque de 1876-1878, il est nécessaire de tenir compte de ces récits. Ilya PLATOV souligne la singularité inédite de cette guerre qui se manifeste dans la mobilisation politique des masses, sans effort de propagande de la part de l'État impérial. Il montre comment cette guerre est imaginée, anticipée et acceptée par la population qui, par là même, s'impose en tant qu'acteur historique. Cette croisade slave s'inscrit dans un «clair-obscur » idéologique, selon l'acception d'Alphonse Dupront ${ }^{5}$ : à la fois libération chrétienne, donc croisade " contre » l'ennemi tel qu'il apparait à travers les récits et protection de la victime qui partage la même religion et les mêmes conceptions du monde, et croisade "pour » la 
libération des nationalités, c'est-à-dire pour la liberté, la civilisation, la foi et une quête de régénération nationale, héritage de l'universel révolutionnaire, libéral.

Silviu PETCU, à travers l'analyse de La Ţiganiada (épopée placée dans la Munténie du $\mathrm{XV}^{\mathrm{e}}$ siècle) d'Ion Budai-Deleanu, un des pères fondateurs de la langue roumaine moderne, s'arrête sur la lutte de la petite armée roumaine formée de paysans et boyards commandée par le prince Vlad l'Empaleur, surnommé Dracula, contre l'avancée des Turcs vers l'Europe centrale. Ce rempart humain, pour l'historiographie roumaine, aurait permis à l'Europe d'avoir le temps de se préparer et de s'équiper avec des armements plus modernes pour livrer bataille aux Ottomans. L'image négative des Turcs dans l'œuvre rappelle celle qui est largement répandue dans les autres littératures balkaniques, toutefois l'auteur n'épargne pas les boyards, jusqu'au frère de Vlad, Radu le Bel, homme du sultan, qui, sans compassion, usent tout autant de cruauté, perfidie et corruption. Cette épopée met en scène, sur un deuxième plan, les «Tsiganes » mobilisés pour combattre aux côtés de Vlad l'Empaleur avec la promesse d'émancipation des Rroms esclaves et l'attribution d'un territoire en échange de leur alliance. Bien qu'anachronique (l'abolition aura lieu le 20 février 1856), cette idée révèle l'esprit précurseur de Budai-Deleanu.

11 Quant aux Rroms de Roumanie et de Bulgarie, n'ayant produit une littérature écrite que tardivement (après la Seconde Guerre mondiale dans les Balkans), c'est à travers les récits oraux que se dégagent principalement leur vision du monde et leur représentation du Turc. Marcel COURTHIADE, à partir de l'édition d'un corpus de onze chansons rromanies de Bulgarie et une de Roumanie (dont il donne les traductions), étudie les différents noms attribués aux Turcs ainsi que les expressions et locutions turques présentes dans les chansons populaires afin de définir les traits principaux du personnage turc. Le portrait qui se dégage de ces chansons rejoint celui qui domine dans les Balkans, à savoir un Turc impitoyable, féroce et même sadique quand il s'agit de jouer avec la vie et les souffrances de ses victimes.

12 L'image de la période ottomane et la représentation de l'Ottoman, désigné plus généralement «le Turc», ne sont pas figées, elles dépendront de l'époque, de la situation géopolitique du pays et du sort qui est réservé aux minorités, quand les Turcs sont eux-mêmes une minorité. Les variations ainsi que les ruptures dans la continuité d'une représentation sont signifiantes et il est pertinent de tenter d'en comprendre les motivations et les enjeux. Marie VRINAT-NIKOLOV s'interroge sur la permanence de l'image négative de l'Ottoman héritée du passé, dans les œuvres littéraires bulgares de 1878 à la fin du XXe siècle et s'attache à établir une typologie romanesque de l'Ottoman. Si la Bulgarie, par sa situation géographique, son histoire et ses origines, se situe à la lisière entre l'Orient et l'Occident, ce n'est que très récemment qu'un travail de démythologisation a été entrepris par les historiens, les anthropologues et les critiques littéraires. Dans la prose "postmoderniste » de la jeune génération, Vrinat-Nikolov remarque l'absence du thème ottoman et la mise en place de collaboration dans le domaine littéraire entre auteurs bulgares et turcs, ce qui signe la rupture d'avec la représentation traditionnelle du Turc.

13150 ans de proche cohabitation avec l'Empire ottoman ont laissé de nombreuses traces dans la culture et la littérature hongroises. L'image de l'Empire a subi divers changements au cours des siècles. Outre les rapports militaires qui ne relatent que des attaques surprises menées avec courage ou fanatisme, les premières sources qui présentent des images nuancées sont les écrits des esclaves rentrés au pays après de 
longues années passées dans l'Empire ottoman. Le dominicain transylvain Georgius de Hungaria écrira en 1470 un traité sur l'Empire où domine une vision positive des Ottomans allant jusqu'à considérer leurs valeurs morales comme exemplaires. Le siècle suivant, Hans Dernschwam, Saxon de Bohème, inverse le jugement. Dans son Itinéraire de Turquie (datant de 1553-1555), il ne voit dans l'Empire que «bizarreries ridicules et vicieuses ». Parallèlement, Sebastian Tinódi, dit le Ménestrel, dans son recueil de chansons de geste Cronica, dépeint le sultan Soliman, comme un ennemi redouté par les puissances occidentales. Au XVII ${ }^{e}$ siècle, Miklos Zrinyi, chef militaire qui se bat avec succès contre le Turc, dans son épopée Le péril de Sziget, reconnait l'héroïsme et la vaillance des preux Ottomans. Il fait de Soliman une figure légendaire. Le XIX inaugure deux nouvelles voies : le romantisme avec Mór Jókai qui peint une âme profonde et orientale et le réalisme de Tömörkény qui s'intéresse aux petites gens. Le $\mathrm{XX}^{\mathrm{e}}$ siècle exploite le sujet ottoman tout en hésitant entre l'honorable ennemi et le compagnon de fortune. Dans cette littérature, Györgyi MATE choisit de traiter la question de l'honneur, plus précisément de la parole donnée, sur trois axes: les engagements personnels (politiques et militaires), les conversions et le fonctionnement de la cohabitation.

Quant aux Serbes, avec l'arrivée des Ottomans et malgré la perte de leur Empire, leur imaginaire collectif continue à créer leur portrait historique à travers la poésie épique. Dans la présence ottomane, les Serbes ne voyaient pas seulement l'envahisseur impitoyable, mais surtout leur propre échec, explique Sanja BOŠKOVIĆ. D'où une poésie épique sur un thème unique: la reconquête de la liberté. La poésie orale étant l'expression collective d'une représentation sublimée de l'identité, le portrait historique des Serbes est en tous points positif. Cependant, lorsque le Turc égale, voire surpasse, en bravoure le héros serbe, il mérite respect, honneur et crainte, selon les valeurs propres à la morale hérö̈que des sociétés traditionnelles.

La littérature peut-elle être réduite à la réalité historique à laquelle elle se réfère ? Branka ŠARANČIĆ met en évidence le potentiel subversif de la philosophie romanesque d'Ivo Andrić (prix Nobel 1961) qui remet en question la notion de "vérité historique " et définit le travail de l'écrivain comme une traduction de l'Histoire en une multitude d'histoires s'attachant davantage à ce qui est hors de portée de l'Histoire, mais qui répond à la quête d'un peuple à la recherche de son identité. La recherche du sens caché de l'histoire collective n'étant pas motivée par la défense d'une cause nationale ou politique, mais par la défense de l'autonomie intellectuelle de l'artiste, nous avons de multiples images contrastées de l'Empire ottoman, de même qu'une représentation sur plusieurs registres : mythique, poétique, comique, tragique et morbide de l'Orient. L'univers romanesque de l'écrivain bosniaque Meša Selimović échappe à la codification traditionnelle des genres littéraires, même s'il emprunte pour cadre historique la période ottomane. Selon Branka ŠARANČIĆ, les romans de Selimović contribuent à redéfinir le statut de la littérature dans son rapport au politique, et plus précisément celui de l'auteur dont l'indépendance intellectuelle doit rester un préalable incontournable pour trouver des solutions inédites sur les plans de la création littéraire et des représentations identitaires.

17 L'Empire ottoman peut servir de cadre pour une critique des sociétés contemporaines. C'est alors l'aspect despotique, tyrannique et destructeur qui sera mis en avant. Ceux qui, au sortir de l'Empire, ont pensé avoir été trahis, reprochent alors aux Ottomans d'avoir voulu les éloigner de l'Europe à laquelle ils pensaient appartenir. Jean Paul 
CHAMPSEIX voit dans les œuvres antiutopiques d'Ismaïl Kadaré une condamnation du communisme vu comme un avatar du despotisme asiatique, voire de toute volonté de pouvoir absolu.

18 La Bosnie, appelée l'obscur vilayet, sous occupation ottomane du XV $\mathrm{XV}^{\mathrm{e}}$ XIX ${ }^{\mathrm{e}}$ siècle, possède une littérature qui a conservé de nombreuses traces de cette période. Dans ce pays qui se caractérise par la diversité de sa population, les Slaves convertis à l'islam rédigeaient leurs œuvres en langues orientales (turc, arabe et persan); leurs recueils de poésie, divan, écrits en turc ottoman, sont imprégnés de la pensée soufie (mystique musulmane), un phénomène caractéristique de la Bosnie, précise Jasna ŠAMIĆ. La littérature dite alhamiado ou aljamiado regroupe les auteurs qui s'exprimaient aussi en langue slave, bosniaque, en utilisant les caractères arabes. Un autre genre représentatif de l'art populaire musulman, essentiellement urbain, la sevdalinka est très connu de nos jours dans tous les Balkans. À la fin du XIX ${ }^{\mathrm{e}}$ siècle apparaît le courant des auteurs qui écrivent en langue bosniaque, en utilisant les caractères latins, tel Safvet Bey Bašagić (1870-1934) ou Musa Ćazim Ćatić (1878-1915) dont la poésie témoigne du mélange des deux cultures, tout en restant un vestige ottoman. Ce mélange des cultures est visible aussi dans les ouvrages des chrétiens de Bosnie, surtout dans la poésie d'Aleksa Šantić (1868-1924). La guerre en Bosnie (1992-1995) marque une rupture et supplante la thématique ottomane dans les œuvres contemporaines. Cependant, les auteurs sont toujours inspirés par la religion musulmane, voire le soufisme, constituant l'illustration selon laquelle la thématique ottomane est plus qu'une inspiration, elle est un élément culturel et identitaire des Bosniaques.

19 L'Empire ottoman a donné naissance à plusieurs "questions ». La question d'Orient dont le pivot était la Macédoine et la question arménienne. Le partage de la Macédoine entre quatre États (Grèce, Serbie, Bulgarie et Albanie) en 1913 dispersa le peuple macédonien de telle sorte qu'il lui était difficile d'asseoir sa légitimité et sa reconnaissance. L'insurrection d'llinden (Saint Élie) en 1903, à l'initiative des révolutionnaires macédoniens, dont l'aboutissement est la création de la première République démocratique des Balkans: la République de Kruševo, inspirera de nombreuses créations littéraires macédoniennes, en particulier le théâtre. Despina ANGELOVSKA s'attache à analyser deux pièces : Ilinden de Nikola Kirov Majski, acteur dans l'insurrection et auteur du Manifeste de Kruševo (dont nous donnons la traduction intégrale) et Le fantôme de la liberté de Vojdan Černodrinski, écrites quelques années après l'insurrection. À travers ces créations produites avec une distance temporelle par rapport à l'événement, il s'agit de porter un regard critique sur les représentations de l'Histoire et les discours idéologiques que soutiennent, renforcent ou déconstruisent ces œuvres littéraires pour permettre l'existence d'autres narrations. C'est aussi, à travers la relecture de certains événements historiques, un questionnement sur l'actualité de la situation macédonienne. Le Manifeste de Kruševo, dans le respect de la diversité des peuples et de leurs identités, de leur égalité en droit, n'appelle-t-il pas à s'opposer à tout réductionnisme nationaliste? N'enseigne-t-il pas le partage? Si les peuples opprimés ont pu partager l'injustice, la misère, la souffrance, pourquoi, libres, ne pourraient-ils pas partager une République?

Pour Vlada UROSEVIĆ, par sa lecture du roman Comment tuer Apostol? de l'écrivain macédonien Slobodan Micković (1935-2002), une réconciliation entre oppresseurs et opprimés était possible dans un pays réformé où les citoyens auraient été égaux en droit, mais elle a été manquée. La multiethnicité aurait pu perdurer à condition que les 
anciennes rancunes soient oubliées. Or, malgré les idées progressistes des protagonistes, l'obsession du passé et des antagonismes empêchent l'émergence de sociétés démocratiques fondées sur le respect de la diversité des peuples et des cultures. Ce roman paru en 1994, qui a pour cadre l'Empire ottoman de 1908, lorsqu'anciens et jeunes Turcs s'opposent dans leur conception de l'État, rompt avec l'image stéréotypée de l'Empire ottoman comme période historique où règnent le despotisme asiatique, l'obscurantisme et la corruption.

Les communautés diasporiques des Balkans ont été très peu étudiées, toutes disciplines confondues. La littérature née en diaspora, souvent négligée, est rarement intégrée dans l'histoire générale de la littérature du peuple concerné. Les œuvres ne sont, bien souvent, ni cataloguées, ni étudiées. Ces productions pâtissent de leur statut de produit d'une expérience de confrontation avec un nouveau pays. Pourtant, dans les Balkans, il y eut de nombreuses émigrations, migrations et déplacements de populations. L'étude de Krikor BELEDIAN a pour objet la monumentale monographie de Hagop Sirouni, alias Djololian (Adapazar 1890-Bucarest 1973) : Constantinople et son rôle, parue en quatre volumes, d'environ 2000 pages et restée inachevée. Ce poète, prosateur, théoricien de la littérature et historien arménien, exilé en Roumanie en 1923, arrêté en 1944 et condamné à dix ans de camp sibérien, est obsédé par la nostalgie d'un pays perdu et hanté par la Catastrophe (génocide) de 1915. À l'instar de tout rescapé, il fait de l'art une modalité de la survie. Pour la rédaction de cette œuvre, commencée en 1947, Sirouni mettra à contribution aussi bien des informations contenues chez les voyageurs européens que des renseignements fournis par les sources ottomanes et par les écrivains arméniens $d u$ XVII $^{\mathrm{e}}$ et XVIII ${ }^{\mathrm{e}}$ siècles. Le projet, empreint d'une visée encyclopédique, envisage Constantinople comme une totalité, il s'agit de réaliser une synthèse de cinq siècles d'histoire (des Arméniens et des Ottomans), de culture, de littérature et d'art. Vers la fin du quatrième volume, le lecteur mesure la crise à laquelle est confronté l'auteur en tant que contemporain et témoin des événements historiques qu'il relate. L'inachèvement de l'œuvre en est-elle la conséquence? Beledian note un certain renoncement progressif au récit littéraire pour le récit historique, dans l'écriture de Sirouni. L'Empire ottoman demeure néanmoins l'objet désiré dont l'image fascine et déchire l'exilé tout au long de son exil.

Quelques textes rares et non traduits de la littérature romanesque grecque du $\mathrm{XIX}^{\mathrm{e}}$ siècle ont pour thématique la ville de Constantinople. À cette époque les romanciers grecs, rivalisant avec leurs confrères français, précise Henry TONNET, cherchent un cadre urbain comparable au Paris d'Eugène Sue ou de Balzac pour situer leurs intrigues. Constantinople apparaît comme la capitale naturelle de l'hellénisme. Grecs riches et pauvres de la ville occuperont, de manière exclusive, le devant de la scène romanesque. Le point commun de tous les romans de cette période est qu'ils soulignent la continuité entre l'Empire Byzantin et l'Empire ottoman. Bien que la ville imaginaire tienne plus de place que la ville réelle, tout au long de ces pages se révèle une Constantinople disparue dont la beauté fascine les auteurs. Les Turcs sont, pour ainsi dire, absents, pourtant, avant le Désastre d'Asie Mineure de 1922 et les événements de 1955 qui entraînèrent le départ de la quasi-totalité de la colonie grecque d'Istanbul, les Grecs pensaient possible une entente, car ils tenaient les Turcs moins pour des êtres féroces que primitifs et naïfs, d'où cette fameuse "simplicité ottomane " (aplotis Othomaniki). 

romanesques de la littérature grecque situent leur action à l'époque de l'Empire ottoman. Nicolas PITSOS a choisi de nous présenter le roman de Vassilis Christopoulos (2005), Toi aussi, tu es Grec? qui participe de cette tendance littéraire. Le roman est construit sur l'histoire de plusieurs familles dont l'existence se déroule lors de l'effondrement de la société ottomane qui repose sur le principe des millets - fondé sur l'identification des individus en fonction de leurs appartenances religieuses - et le passage à une nouvelle organisation politique : l'État nation qui définit l'individu sur la base de critères nationaux. Il s'intéresse particulièrement au passage du millet des Roums à la nation des Grecs. La narration se fait à plusieurs voix, expressions d'expériences traumatisantes de politiques de nettoyage ethnique et culturel qui agitent l'Empire ottoman à cette époque, émanant d'individus issus de peuples différents. L'intérêt et l'originalité de ce roman résident dans cette écriture plurielle et, par là même, dissidente, qui s'oppose à une perception exclusiviste d'un événement historique ainsi qu'à sa transformation en mythe national visant à exalter les souffrances des compatriotes et à monopoliser le rôle de victime tout en effaçant ou occultant les destins tragiques des autres populations. Ce «nouveau roman historique " se démarque du roman historique du XIX ${ }^{e}$ siècle, élaboré selon la théorie narrative de Walter Scott, par sa focalisation sur le processus conflictuel de la mise en place d'une mythologie nationale servant à légitimer l'existence de l'État-nation et à assurer son unité.

Quelles images revêt l'Ottoman dans la littérature des Juifs expulsés d'Espagne en 1492 et réfugiés dans l'Empire ottoman. Marie-Christine VAROL analyse l'autobiographie romancée d'Eliya Karmona publiée en 1926: Comment naquit Eliya Karmona comment il grandit et comment il devint directeur du Djugetón. Ce roman picaresque, sur le mode sarcastique, nous livre un regard critique sur les tribulations d'un jeune homme juif dans les dernières années du sultanat (1884-1908) ainsi qu'une critique acerbe et comique des pesanteurs du système ottoman, qui font obstacle à toute initiative, et des raideurs communautaires qui pèsent comme un carcan sur l'initiative personnelle. Cependant, l'œuvre révèle en creux une admiration pour certaines valeurs ottomanes (en particulier aristocratiques : les usages de la politesse, le raffinement des mœurs, le savoir-vivre, l'élégance et le bon goût) et une certaine nostalgie pour ce monde ancien disparu que, plus encore que les Juifs, les notables turcs représentent. Nostalgie qui, avec la disparition de l'Empire ottoman et en regard du génocide dont ils ont été victimes, est partagée par les communautés juives.

Mustafa Kemal Atatürk (1881-1938), en fondant la République turque, instaure une rupture avec l'Empire ottoman et fait table rase du passé ottoman. Il nous a semblé important d'étudier les images de la période ottomane dans les œuvres des écrivains turcs contemporains. Le roman turc du début du $\mathrm{XX}^{\mathrm{e}}$ siècle traite de la mort de l'Empire et glorifie l'avènement de la République dans sa vision kémaliste moderniste et occidentaliste. Progressivement, cependant, des écrivains, désireux de connaître le passé controversé, prennent pour sujet le début de la fondation de l'Empire ottoman. Les origines des Ottomans et leur histoire jusqu'au XIV siècle ont été longtemps méconnues, aussi est-ce un sujet qui suscite des débats académiques souvent enflammés. Valérie GAY, choisit d'étudier deux écrivains : Kemal Tahir et Tarik Buğra, dont les romans Devlet Ana (La mère Patrie, 1967) et Osmanclk (Le petit Osman, 1983) ont pour sujet le début de la fondation d'un ordre étatique par les tribus turkmènes aux 
$\mathrm{XIII}^{\mathrm{e}}$ et XIV ${ }^{\mathrm{e}}$ siècles. Ces œuvres ont été reçues à la fois avec un vif enthousiasme et de violentes critiques. Le débat historiographique, la manière dont il se reflète dans le roman turc et le contexte politique et culturel dans lequel ont vécu les deux auteurs sont les trois aspects à travers lesquels seront traitées ces œuvres par Valérie Gay.

Elle montre que, bien que distincts, ces deux auteurs, l'un marxiste (Kemal Tahir), l'autre défenseur de l'Islam (Tarik Buğra), se rejoignent dans la même opposition aux tabous historiques officialisés par le régime républicain, le même recours à des modèles formels relevant du mythe, le même enthousiasme pour l'époque ottomane, ce qui les conduit à une même défense des valeurs ottomanes et de la culture nationale contre les courants d'occidentalisation. Kemal Tahir allant jusqu'à qualifier le concept d'humanisme, tel que pensé par l'Occident, de "rideau de fumée " servant à « dissimuler l'exploitation bourgeoise », quant à l'occidental, il serait « un cannibale en cravate qui, une fois qu'il a décidé de dévorer de la chair, ressemble, pour ceux qui regardent de l'extérieur, à l'église chrétienne qui embrasse les gens en leur faisant lever la tête, mais pour ceux qui voient l'affaire de l'intérieur, il est clair que c'est à coups de pied qu'on leur fait redresser la tête'. ${ }^{6}$ Le rejet des Orientaux par les Occidentaux n'entraîne-t-il pas immanquablement le rejet des Occidentaux par les Orientaux! Dans un miroir à double face, le clair et l'obscur se reflètent inversement.

\section{NOTES}

1. «[...] pour le début du XVI ${ }^{\mathrm{e}}$ siècle cinq Voyage à Constantinople alors que, après 1520 , de l'avènement de Soliman jusqu'à la fin du siècle plus de cent cinquante récits de voyage sont publiés (avec un accroissement considérable dans le dernier quart du XVI ${ }^{\mathrm{e}}$ siècle); il y a là manifestement un immense mouvement de curiosité, un vif désir de savoir, de connaître, à mettre en rapport avec l'élan intellectuel, artistique, l'ouverture au monde que représente la Renaissance en Occident. Si beaucoup de ces relations ne sont que des descriptions, parfois sommaires, de la capitale, des évocations du voyage ou des conditions de vie, quelques-unes mettent en lumière les caractères du gouvernement ottoman, s'attardent sur les principes religieux musulmans; et bien souvent ce ne sont pas des critiques qu'expriment leurs auteurs, mais de l'émerveillement, de l'étonnement et des louanges. Au régime de Soliman, même s'il n'atteint pas la perfection, ils reconnaissent des mérites et qu'il sait en particulier faire respecter les vertus de loyauté, de tolérance au moment même où l'Occident est ravagé par des guerres civiles et religieuses. L'exemple donné de l'Empire du Grand Turc et de la vie paisible de sa capitale est parfois une manière déguisée de critiquer les gouvernements occidentaux, sans réelle grandeur.

La multiplication des voyages d'Occidentaux et de leurs relations écrites traduit la curiosité et l'admiration de l'Europe entière pour le sultan, pour tout ce qui l'entoure, pour son système de gouvernement. Le prestige de l'Istanbul de Soliman est alors si grand qu'il ferait presque oublier le souvenir de la Constantinople byzantine. C'est là un sentiment commun à tous les voyageurs : «La ville de Constantinople est située en ce lieu le mieux à propos pour la grandeur d'un 
souverain que nulle autre ville au monde ", écrit le Français Pierre Belon qui séjourna dans la cité au XVI ${ }^{\mathrm{e}}$ siècle. » Robert Mantran, Histoire d'Istanbul, Fayard, 1996, p. 243.

2. M. Leuridan, Le « Janissaire » et le " Pianoforte », in La revue de l'Association Charles Plisnier asbl, Francophonie vivante, septembre 2007. Cf. aussi : http://www.charles-plisnier.be/

3. Ibidem.

4. Ibidem.

5. A. Dupront, Le mythe de croisade, Paris : Gallimard, 1997, t. 1, p. 533 ; t. 2, p. 933.

6. Selim İleri, “Kemal Tahir'le Konuşma”, Yeni Dergi, Juin 1973, p. 105, cité par Valérie Gay, voir son article dans ce numéro. 